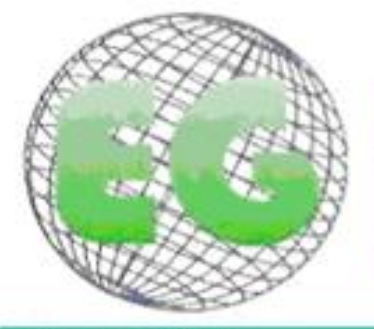

\title{
Métodos de enseñanza utilizados por docentes del curso de enfermería: enfoque en la metodología de investigación
}

Metodologias de ensino utilizadas por docentes do curso de enfermagem: enfoque na metodologia problematizadora

Teaching methods used by teachers of nursing: focus on questionable methodology

\section{${ }^{*}$ Paim, Aramasi Silva ${ }^{*}$ lappe, Nadine Thauana ${ }^{* *}$ Rocha, Daniele Laís Brandalize}

*Graduada en Enfermería por la Pontifícia Universidade Católica do Paraná (PUCPR). E-mail: aramasipaim@hotmail.com **Máster en Enfermería. Docente del Curso de Graduación en Enfermería PUCPR. Brasil.

\author{
Palabras Clave: Educación superior; Enfermería; Métodos \\ Palavras chave: Educação superior; Enfermagem; Métodos \\ Keywords: Education; higher; Nursing; Methods
}

\section{RESUMEN}

Objetivos: Contribuir al desempeño de los docentes de enfermería a través de metodología cuestionable e identificar los métodos de enseñanza utilizados por el profesorado de la asignatura Enfermería y presentar la metodología de investigación de los profesores.

Métodos: La investigación cualitativa, el método exploratorio y descriptivo, mediante entrevistas semi estructuradas con 14 profesores de enfermería.

Resultados: Un análisis de contenido de Bardin reveló seis unidades de contexto y trece unidades de significado que mostraron las diferentes metodologías utilizadas por los profesores y aprender de ellos acerca de ellos. Durante la presentación de la metodología cuestionable llevó a discutir otros métodos utilizados, después de haber sido una reunión productiva y que puedan contribuir a la práctica docente.

Conclusión: Llegamos a la conclusión de que debería haber más reuniones de discusión sobre las metodologías de enseñanza.

\section{RESUMO}

Objetivos: Contribuir na atuação dos docentes do curso de enfermagem através da metodologia problematizadora e identificar as metodologias de ensino utilizadas pelo docente do curso de Enfermagem e Apresentar a metodologia problematizadora aos docentes. 
Médotos: Pesquisa qualitativa, método exploratório-descritivo, utilizando a entrevista semi-estruturada com 14 docentes do curso de enfermagem.

Resultados: A Análise de Conteúdo de Bardin revelou seis Unidades de Contexto e treze Unidades de Significação que mostraram as diferentes metodologias utilizadas pelos docentes e o conhecimento dos mesmos sobre elas. Durante a apresentação da metodologia problematizadora aproveitou-se para discutir as demais metodologias utilizadas, tendo sido um encontro produtivo e que pode contribuir para a atuação docente.

Conclusão: Conclui-se que deve haver mais encontros de discussão sobre as metodologias de ensino.

\section{ABSTRACT}

Objectives: To contribute to the performance of teachers of nursing through questionable methodology and identify the teaching methods used by the faculty of the Nursing course and present the investigative methodology to teachers.

Methods: Qualitative research, exploratory and descriptive method, using semi-structured interviews with 14 teachers of nursing.

Results: A content analysis of Bardin revealed six units of context and thirteen units of meaning which showed the different methodologies used by teachers and learn about them about them. During the presentation of the questionable methodology took to discuss other methods used, having been a productive meeting and that can contribute to the teaching practice.

Conclusion: We conclude that there should be more discussion meetings on teaching methodologies.

\section{INTRODUCCIÓN}

Se observa, actualmente, un gran aumento en la oferta de educación superior en diversas áreas del conocimiento. Como censo realizado por el Ministerio de Educación, en 2010, el número de matrículas en los programas de pregrado se ha incrementado en un $7,1 \%$ de 2009 a 2010 y $110,1 \%$ entre 2001 y 2010 . Este fenómeno trae consigo muchos puntos para la discusión y reflexión, entre ellos la formación profesional para la enseñanza en la educación superior ${ }^{(1)}$.

A juicio de las autoras, lo que se encuentra, a menudo, es que profesionales entran en el campo profesional de la educación superior por varias razones y no tienen una formación actual y complementaria para trabajar como profesor, así al no tener esa formación terminan repitiendo la misma manera tradicional de enseñanza que se presentaron, sin existir preparación y actualización para cumplir con las nuevas necesidades profesionales requeridas por el mercado laboral.

Actualmente, la enseñanza se define como las actividades educativas que constituyen el proceso de enseñanza y aprendizaje y que tienen la responsabilidad de fomentar la creación cultural, el desarrollo del espíritu científico y del pensamiento reflexivo y crear condiciones favorables para el desarrollo de los estudiantes en diferentes áreas del conocimiento, en el aspecto afectivo-emocional, en las habilidades y en las actitudes y valores ${ }^{(2)}$.

Sin embargo, para llevar a cabo esta actividad educativa como un proceso de enseñanza y aprendizaje que resulte en la producción y la construcción del conocimiento, las instituciones de educación superior, junto con sus profesores, necesitan abandonar la tradicional postura, aulista y expositiva de transmitir las ideas, para tratar de renovar las metodologías que están obsoletas como la exposición por 
parte del profesor y, finalmente, tomar posesión de la perspectiva educativa moderna, asociada con la era de la sociedad del conocimiento ${ }^{(2)}$.

En este sentido, se cae en la cuenta de la necesidad de transformaciones en la educación de enfermería, ya que los estudiantes deben participar como sujetos en la construcción y reconstrucción del conocimiento y no sólo como espectadores y receptores de la información. Este cambio de paradigma educativo contribuye a que se tenga un profesional crítico, reflexivo y capaz de proponer cambios y soluciones a los problemas experimentados en su práctica profesional.

No obstante, esto sólo es posible si los educadores remodelan su visión del proceso de enseñanza y aprendizaje, pasando de transmisor a facilitador de este proceso, en el que el estudiante, no el profesor, es el actor principal, lo que permitirá el desarrollo efectivo del espíritu crítico, es decir, que permitan a los estudiantes desarrollar su capacidad de cuestionar, investigar, diverger, discutir, analizar, probar y evaluar ${ }^{(3)}$.

Lo que llevó a este estudio fue la percepción de las autoras, que son estudiantes de enfermería, del uso extensivo de las presentaciones orales de los profesores como metodología de enseñanza, con pocos cambios en otras metodologías, que a veces desmotivan a los estudiantes, ya que a menudo, no se les anima a buscar el conocimiento más allá de lo que el profesor enseña en el aula.

Por tanto, el presente estudio tuvo como objetivo general contribuir a la actuación de los profesores del curso de enfermería a través de la educación problematizadora, e identificar los métodos de enseñanza utilizados por los profesores de enfermería y presentarle la metodología problematizadora.

Se propuso esta investigación por considerar que es un tema de gran relevancia para la enseñanza y la práctica en materia de salud y de enfermería, ya que, mediante la identificación de los métodos de enseñanza utilizados por el profesor y la presentación de la metodología problematizadora a los profesores, se podrá estimular la reflexión de cada uno de los docentes sobre la idoneidad de las metodologías que utilizan en sus clases, para mejorar su práctica con la consecuente educación de calidad y actual en el curso de enfermería.

Basándose en lo anterior, se propone como pregunta guía de este trabajo: ¿En qué medida la educación problematizadora puede contribuir a la actuación de los profesores del curso de enfermería?".

\section{JUSTIFICACIÓN TEÓRICA}

Sabemos, ahora, que el aprendizaje se produce cuando los estudiantes participan activamente en la construcción y reconstrucción del proceso de conocimiento. En esta perspectiva, hay que sustituir, en las clases, las tareas mecánicas que requieren la repetición y la memorización, por tareas que requieren de los estudiantes la realización de operaciones mentales ${ }^{(4)}$.

Para lograr que este nuevo enfoque de la enseñanza sea alcanzado, los profesionales enfermeros que trabajan o que quieran trabajar como profesores en la educación superior necesitan conocer los métodos y técnicas de enseñanza y aprendizaje, así podrán utilizarlos de manera apropiada a cada situación de enseñanza y aprendizaje. 
La metodología didáctica se define como un conjunto de métodos y técnicas de enseñanza para el aprendizaje. El método es el camino para alcanzar una meta, la técnica es el proceso de hacer algo, cómo hacerlo. La elección de la metodología de enseñanza y aprendizaje se lleva a cabo de conformidad con las condiciones y circunstancias del estudiante, del maestro y del medio ambiente ${ }^{(5)}$.

Así, los métodos de enseñanza se estructuran en torno a las técnicas de enseñanza que pueden ser entendidas como el conjunto de procesos de un arte, habilidad o forma de realizar algo. Según Vilarinho ${ }^{(6)}$, los métodos de enseñanza se presentan en tres modos básicos: métodos de enseñanza individualizada, métodos socializados y métodos socio-individualizados. Y Libâneo apud Evangelista y Chaves ${ }^{(7: 62)}$, añade uno más, modo básico de la enseñanza, la Exposición de contenidos por parte del profesor, o sea, el método de enseñanza expositivo dialogado.

El método de enseñanza individualizado se basa en tareas de estudio dirigidas, impulsadas por el profesor a los estudiantes. En el método socializado, el foco principal es el trabajo en grupo, la integración con los medios sociales y este método tiene como objetivo desarrollar la capacidad de interacción interpersonal para que los estudiantes aprendan a expresar y defender sus ideas. Son ejemplos de la educación socializada los estudios de caso, grupos de discusión, seminarios y conferencias.

El método de enseñanza socio-individualizado es la interacción entre profesores y estudiantes que tiene como fin producir nuevos conocimientos, habilidades, la reflexión acerca de ellos. Su aplicación está condicionada al conocimiento de los estudiantes de los objetivos previstos, el dominio de los conocimientos básicos y la disponibilidad de adquirir y producir nuevo conocimiento y tiene como ejemplo el método de resolución de problemas y la investigación como actividad de los estudiantes.

En el método de exposición por el docente o expositivo dialogado, el profesor tiene la responsabilidad de la presentación, explicación y demostración de los conocimientos, las habilidades y las tareas. De este modo, el estudiante se convierte en un receptor de conocimientos, pero no pasivamente. Por ser un método ampliamente adoptado, es necesario tener cuidado con su uso, con el fin de evitar prácticas didácticamente incorrectas generalmente percibidas como: el énfasis en la memorización de contenidos sin comprenderlos; el uso de palabras sin correspondencias con el vocabulario conocido por los estudiantes; la falta de relación entre los contenidos; la exigencia de silencio durante la exposición, con el uso de amenazas e intimidades; la adopción de métodos de evaluación que requieren respuestas que, literalmente, reproducen los contenidos transmitidos por el profesor o el libro didáctico; no tener en cuenta las diferencias individuales y la realidad de los estudiantes ${ }^{(7)}$.

Este método de enseñanza se destaca en la enseñanza de la enfermería actual y está muy cerca de la pedagogía tradicional, ya que el énfasis está en la transferencia de conocimiento. En esta tendencia, las acciones de la enseñanza están centradas en la exposición de los contenidos por parte del profesor, el cual supervisa, asesora, enseña y corrige el problema. Se le ve como la máxima autoridad y el único responsable y conductor del proceso educativo. Hay un predominio de la presentación oral, siguiendo una secuencia fija predeterminada y se hace hincapié en la necesidad de ejercicios repetidos para asegurar la retención del contenido, los cuales no están relacionados con la vida cotidiana del estudiante, y mucho menos con las realidades sociales $^{(8)}$. Como resultados se tienen la repetición de conocimientos, la dificultad del 
estudiante para aplicar la teoría en la práctica y poco aprendizaje, ya que gran parte del contenido que se almacena es pronto olvidado.

En oposición a esta pedagogía, se tiene la Pedagogía Crítica, destacando la Metodología de la Problematización, que se reúne a la nueva propuesta de enseñanza y aprendizaje como un método de instrucción socio-individualizado. Esta metodología se revela como una estrategia innovadora en la educación, sea como método de aprendizaje o enseñanza, y se basa en el pensamiento de Freire, lo que favorece el intercambio de conocimientos y experiencias entre los alumnos y el profesor, mientras que ambos cuentan con una sola historia -y colectiva- y un contexto social compartido ${ }^{(9)}$.

En la metodología problematizadora, la educación es una actividad en la que "los profesores y los estudiantes están mediatizados por la realidad que aprenden y desde ella extraer el contenido de aprendizaje, alcanzan un nivel de conciencia de esta realidad con el fin de actuar en ella, lo que permite la transformación social"(8).

Esta metodología permite al estudiante hacer la conexión/enlace entre el conocimiento teórico y la práctica, es decir, de teorizar los problemas y/o situaciones reales de la vida del día a día o del mundo que le rodea. El profesor facilita el desarrollo libre y espontáneo de los estudiantes ${ }^{(8)}$.

El proceso que compone esta metodología se divide en cinco fases y comienza con la observación de la realidad, en la que a través de la observación del problema, los estudiantes expresan sus opiniones personales en relación a él e identifican sus puntos clave, es decir, lo que es realmente importante en la determinación de la realidad observada. A partir de preguntas sobre cada punto clave, los estudiantes buscan explicaciones teóricas sobre el tema - la teoría, en la que la participación del profesor es crucial. En la cuarta etapa, los alumnos deben enfrentarse a la teoría con su realidad y formular posibles soluciones. Después de revisar los casos, el último paso consiste en aplicar a la realidad las estrategias elegidas ${ }^{(10)}$.

El uso de esta metodología de enseñanza ocurre con la participación activa de los estudiantes, pues el descubrimiento de los puntos clave del problema y la búsqueda de soluciones se hace por ellos, con la mediación del profesor, que reúne la teoría y lleva a los estudiantes a la reflexión y abstracción para llegar a posibles soluciones y conseguir aplicarlas en diferentes contextos de la realidad.

De este modo, el profesor pasa de ser un mero transmisor a un mediador, y el alumno de un simple receptor a un agente activo y co-responsable por la construcción del propio conocimiento, que perdura y trasciende el aula.

\section{METODOLOGÍA}

Para este estudio se optó por la investigación cualitativa, a través del método exploratorio y descriptivo.

La investigación cualitativa tiene como objetivo "traducir y expresar los sentimientos de los fenómenos del mundo social, es reducir la distancia entre el indicador y lo indicado, entre las teorías y los datos, entre el contexto y la acción"(11:1). El método exploratorio realiza descripciones precisas de la situación y quiere descubrir las relaciones entre sus elementos y componentes; este tipo de investigación requiere 
una planificación muy flexible para permitir la consideración de diversos aspectos de un problema o situación ${ }^{(12)}$. Así, la elección de este método de investigación es adecuada para la consecución de los objetivos inicialmente propuestos

Los sujetos de este estudio fueron 14 profesores del curso de pregrado en Enfermería de una universidad privada en el estado de Paraná, y los siguientes fueron los criterios de inclusión: ser profesor de Enfermería y aceptar participar de la investigación. Se decidió entrevistar a profesores de enfermería debido al hecho de ser este público el adecuado para alcanzar los objetivos establecidos.

El método de recolección de datos para lograr el primer objetivo fue una entrevista grabada semiestructurada con preguntas abiertas, en las que el sujeto tiene la oportunidad de discutir el tema investigado. La entrevista fue apropiada para este estudio, ya que permitió la libre expresión de las ideas, pues, a pesar de que fueron seguidas una serie de preguntas predefinidas, estas se realizaron en un contexto muy similar a una conversación informal ${ }^{(13)}$.

En la primera parte del instrumento, se recogieron datos sociodemográficos tales como las iniciales del nombre, el género, la edad, la formación, tipo de formación para la enseñanza, cuanto tiempo trabajan como profesor y la experiencia de trabajo como docente en esta institución. En la segunda parte, también se recopiló información sobre el uso de los métodos de enseñanza en el aula.

Se llevaron a cabo las entrevistas y registros en junio de 2013 y se celebraron reuniones en un lugar que permitiese la intimidad de las personas. En el desarrollo de la investigación fueron respetados los aspectos éticos de la investigación con seres humanos, de conformidad con la Resolución 466/2012, del Consejo Nacional de Salud.

Para entender el significado de los discursos, se utilizó el Análisis de Contenido de Bardin. Este análisis permite, por su técnica, ir más allá de las apariencias y descubrir el verdadero significado de los discursos sin dejar de lado el rigor científico ${ }^{(14)}$. Para el análisis de contenido, fueron seguidos los pasos: 1) Pre-análisis, en el cual se transcribe el material, se hace una lectura flotante y después se delimita lo que será analizado; 2) Exploración del material, con la definición de las categorías, en las que se organizó el material en Unidades de Contexto Significación. Esta fue la fase de la descripción analítica, en la que el material se sometió a un estudio más profundo; 3 ) Tratamiento de los resultados e interpretación, donde los resultados brutos fueron tratados con el fin de llegar a ser significativos. Fue realizada la condensación de las informaciones para el análisis, lo que resultó en la interpretación inferencial, utilizando la intuición, el análisis reflexivo y la crítica ${ }^{(15)}$.

Para lograr el segundo objetivo específico, previamente fue programada una reunión con los profesores que participaron en la encuesta, después del análisis de datos, lo que será descrito más adelante en los resultados.

\section{RESULTADOS Y DISCUSIÓN}

Con respecto a los datos socio-demográficos, la edad de los sujetos varió desde 27 hasta 58 años. En cuanto a la formación profesional, siete eran enfermeras; dos farmacéuticos; dos veterinarios; dos filósofos y un biólogo; el tiempo de trabajo como profesor osciló entre 2 y 18 años. 
Sobre la base de las preguntas de investigación y después de un análisis exhaustivo del contenido de las expresiones de los sujetos se llegó a seis Unidades de Contexto y trece Unidades de Significación, tal como se presentan en la Tabla 1.

Tabla 1. Unidades de Contexto y Significación

\section{UNIDAD DE CONTEXTO}

A) Metodología de enseñanza utilizada: modalidad individualizada

B) Metodología de enseñanza utilizada: modalidad socializada

C) Metodología de enseñanza utilizada: modalidad socio-individualizada

\section{UNIDAD DE SIGNIFICACIÓN}

1) El estudio dirigido como un estímulo para el pensamiento y la autonomía intelectual.

2) Discusiones en pequeños grupos y estudios de caso como una forma de intercambio de ideas y opiniones, resolución de problemas, búsqueda de información y la toma de decisiones.

3) El seminario y la conferencia como una forma de estimular el estudio en profundidad de un tema, la recolección de información y experiencias, la investigación y el conocimiento global de la materia.

4) La visita técnica y la práctica en el laboratorio como un medio para la aplicación de los conceptos teóricos en la práctica.

D) Metodología de enseñanza utilizada: exposición por el profesor o método expositivo

E) Relación de la metodología en la visión del profesor
5) La conferencia dialógica como una manera de mostrar el contenido con la participación activa de los estudiantes.

6) La metodología utilizada por el profesor se considera facilitadora del aprendizaje, relacionando la teoría con la práctica.

7) La metodología utilizada por el docente no contribuye al aprendizaje de los estudiantes cuando ellos tienen dificultades específicas y el profesor no puede proporcionar la atención necesaria a los estudiantes debido a las clases cortas.

8) La metodología utilizada por el profesor es un facilitador del aprendizaje al proporcionar recursos tecnológicos y educativos, tales como multimedia, fotos, videos.

9) La metodología utilizada por el profesor contribuye al aprendizaje de los alumnos cuando el profesor puede relacionar la metodología apropiada para cada tipo de disciplina y clase.

10) La metodología utilizada por el maestro no contribuye al aprendizaje de los estudiantes, pues hace falta una participación más activa del estudiante en el proceso de aprendizaje. 
F) El (des) conocimiento de la metodología problematizadora por los profesores
11) Los docentes conocen la metodología problematizadora de manera fragmentada.

12) Los profesores creen que esta metodología es llevar a cabo estudios de caso en las clases.

13) Los profesores creen que esta metodología se basa en los problemas, que no se utilizan las clases expositivas dialogadas.

Fuente: Elaboración propia, 2013.

La Unidad de Contexto: Metodología de enseñanza utilizada: modalidad individualizada y la Unidad de Significación: estudio dirigido como un estímulo para el pensamiento y la autonomía intelectual, presentan el uso por los profesores de una forma de enseñanza individualizada, el estudio dirigido.

Al utilizar el término "modalidad individualizada" se entiende un método predominantemente individualizado y no exclusivamente, porque hay que destacar que cada lectura, cada reflexión hay un diálogo entre el lector y el autor, una relación entre el estudiante y los textos, libros o materiales educativos, así como también de la persona con sus reflexiones. Por lo tanto, el estudio dirigido encamina al alumno de manera autónoma para su propio aprendizaje, siendo el profesor solamente un orientador de las etapas del proceso ${ }^{(5)}$.

El estudio dirigido es un método o técnica de enseñanza para convertirse en independiente el estudiante en relación al profesor, y tiene dos funciones principales: la primera es de consolidación de los conocimientos a través de una combinación de la explicación del profesor con ejercicios. La segunda es la búsqueda de la solución de problemas a través de preguntas que los estudiantes puedan resolver de manera creativa e independiente ${ }^{(6)}$.

\section{"Buenalección (...) estudio dirigido" (profesor 11). \\ “(...) textos, artículos científicos” (profesor 05). \\ “(...) incentivo mucho la investigación” (profesor 07). \\ “(...) mucha lectura en grupo" (profesor 08).}

La Unidad de Contexto: Metodología de la enseñanza utilizada: modalidad socializada y la Unidad de Significación: Discusiones en pequeños grupos y estudios de caso como una forma de intercambio de ideas y opiniones, resolución de problemas, búsqueda de información y la toma de decisiones, presenta unas formas de metodología en grupos utilizada por los profesores.

La metodología de estudio de caso es una excelente manera de traer un enfoque holístico e interactivo para la enseñanza y el aprendizaje, su principal ventaja es la adopción de un enfoque dirigido a las preguntas y basado en soluciones. Un buen estudio de caso es el vehículo a través del cual una parte de la realidad se pone en el aula $^{(16)}$. 
Las formas tradicionales de enseñanza se basan en clases expositivas. Se supone que el conocimiento es propiedad exclusiva del profesor y, a través de exposiciones, sigue una sola dirección: del docente al alumno. La metodología de los estudios de casos asume que tanto el profesor como el alumno contribuyen al proceso de aprendizaje. A pesar de que el profesor está más familiarizado con el material de un caso que los estudiantes, sus conocimientos no se toman como definitivos, ya que los participantes traen nuevas perspectivas sobre los temas tratados ${ }^{(16)}$.

“(...) presentación de estudios de caso

(...) metodología interactiva" (profesor 06).

“(...) discusiones de caso” (profesor 11).

“(...) estudio de caso, ofrece ejemplos de la práctica” (profesor 02).

La unidad de Significación: el seminario y la conferencia como una forma de estimular el estudio de un tema, la recolección de información y experiencias, la investigación y el conocimiento global de la materia, se presenta una forma de estudio de grupos utilizado por los profesores, pero con mayor profundidad.

El seminario es una forma de trabajo en grupo, muy utilizado en la enseñanza secundaria, pregrado y posgrado. Este "método se construye sobre la base de la enseñanza con la investigación, llevada a cabo en subgrupos, y la discusión de los aspectos investigados, de manera integrada y complementaria, bajo la supervisión del profesor" $^{\prime 17: 70)}$. Con esto, uno se da cuenta de que los estudiantes pueden tomar el lugar del profesor, porque ahora son ellos los que harán una "exposición", explicando un tópico o tema predeterminado, donde puede ocurrir debate o discusión de los puntos clave que se encuentran por la clase.

\section{“(..) conferencias y seminarios” (profesor 01). \\ “(...) utilización de seminarios” (profesor 04). \\ “(..) seminarios, muchos también” (profesor 11).}

Los seminarios y conferencias contribuyen a la construcción de la autonomía del alumno, ya que los estudiantes desarrollan habilidades de investigación en el registro, en la comunicación y en la argumentación oral.

La Unidad de Contexto: Metodología de la enseñanza utilizada: modalidad socioindividualizada y la Unidad de Significación - visita técnica y la práctica en el laboratorio como un medio para la aplicación de los conceptos teóricos en la práctica, presenta algunas de las formas utilizadas por los docentes para que los estudiantes asocian la teoría con la práctica.

La visita técnica es extremadamente importante como herramienta de enseñanza para el profesor, un soporte que ayuda en la realización de las clases, y lo que es más importante, permite al alumno el contacto con la aplicación práctica de los contenidos aprendidos en el aula. 
La visita técnica es una herramienta de gran importancia para la formación académica, ya que permite a los estudiantes mejorar lo que fue aprendido en el aula. Se crea una motivación y expectativa que busca inculcar en el alumno el conocimiento cognitivo posterior a la visita, colaborando con la formación profesional, su concienciación en cuanto al papel profesional en la sociedad, animándole a la actuación ética y responsable, y facilitando la aproximación a la dinámica de la práctica profesional $^{(18)}$.

\section{"(...) visitas técnicas" (profesor 01). \\ "(...) se hace una relación práctica con el laboratorio" (profesor 10).}

La Unidad de Contexto: Metodología de la enseñanza utilizada: exposición por el profesor o método expositivo y la Unidad de Significación: conferencia dialógica como una manera de mostrar el contenido con la participación activa de los estudiantes se presenta destacada entre los métodos de enseñanza utilizados por el profesor en el aula.

La clase expositiva es una de las técnicas de enseñanza individualizada más conocida de la profesión docente, y se basa en una "presentación oral de un tema, por el profesor, y puede tener mayor o menor participación de los estudiantes, en función de los objetivos de la propuesta y de los objetivos de enseñanza "(17) que puede ser la comprensión de un nuevo tema de estudio que trata de presentar ideas, esquemas y de introducción para un mejor rendimiento de los estudiantes. El profesor también podría tener como propuesta la idea de sintetizar el tema, identificando los puntos principales en forma de resumen.

\section{“(..) clase expositiva (con) discusiones y debate” (profesor 06). \\ “(...) exposición dialogada del tema” (profesor 07). \\ “(...) clases expositivas y dialogadas” (profesor 08). \\ “(...) clases expositivas siempre” (profesor 10).}

Según la nueva pedagogía, la práctica de clase expositiva dialógica permite la posibilidad de transformar una clase que simplemente transmitiría conocimientos listos por parte del educador, en una clase donde los estudiantes tendrán la oportunidad de mostrar sus conocimientos previos, asociándolos a las informaciones presentadas por el docente y finalmente tejer una relación entre el conocimiento y la experiencia, lo que permite el surgimiento de preguntas ${ }^{(17)}$.

Teniendo en cuenta todos los métodos anteriores, vale la pena recordar que ninguno de ellos debe ser utilizado como la principal forma de enseñanza, ya que puede ser bastante inadecuado para el aprendizaje, pues el aprendizaje se produce en varios niveles y en varias formas y se pueden lograr excelentes resultados sólo a través de la adopción de distintas metodologías, asegurando así que todos los estudiantes encuentren la forma de aprendizaje que más les convenga.

La Unidad de Contexto: Relación de la metodología en la visión del profesor y la Unidad de Significación: La metodología utilizada por el profesor se considera facilitadora del aprendizaje, al relacionr la teoría con la práctica muestra que algunos 
profesores creen que las metodologías que utilizan contribuyen a que el alumno relacione la teoría con la práctica.

Los docentes reportan que en el curso de Enfermería el proceso de enseñanza y aprendizaje se hace más fácil cuando el estudiante es capaz de relacionar la teoría con la práctica, esto ocurre debido a la naturaleza y características del curso. Se observó que los profesores que creen que su metodología de enseñanza es eficaz para relacionar la teoría con la práctica, utilizan principalmente el método de estudio de caso.

"Sí, porque tengo como objetivo lo de que los estudiantes asocian la teoría a la práctica" (profesor 01).

"Yo creo que sí, porque simulamos la práctica" (profesor 02).

"Mucho, yo creo que sin la experiencia, sin la práctica, no habría una buena formación" (profesor 13).

En cambio, algunos profesores tienen dificultades para transmitir esta relación con el estudiante, sobre todo en las materias básicas en las que los estudiantes no logran comprender la importancia de estas cuestiones y la correlación con su futuro ejercicio profesional.

"(...) para mí es un poco difícil porque yo enseño en el primer año, entonces ellos entran, y muchos ni siquiera saben lo que es el curso, son disciplinas muy básicas para que esta correlación entre la teoría y práctica sea hecha" (profesor 09).

La Unidad de Significación: la metodología utilizada por el docente no contribuye al aprendizaje de los estudiantes cuando ellos tienen dificultades específicas y el profesor no puede proporcionar la atención necesaria a los estudiantes debido a las clases cortas.

A menudo, el profesor puede utilizar una metodología apropiada para el tema de su clase, puede preparar científicamente en relación con el contenido que va a enseñar, pero el estudiante no es capaz de aprender o seguir el aprendizaje de otros estudiantes. Los informes de los docentes dicen que ellos tienen dificultades para trabajar con este estudiante, ya que no tienen tiempo suficiente para eso.

Para Osti ${ }^{(19: 67)}$, "una de sus principales tareas (del profesor), además de percibir la dificultad de aprendizaje, es solicitar la remisión de proporcionar el diagnóstico y los medios para la atención adecuada". Es decir, el profesor tiene la responsabilidad de detectar dificultades del alumno en el aprendizaje y encaminarlo para que sean tomadas las providencias que contribuyan con el aprendizaje.

"No muy a menudo, los estudiantes tienen algunas dificultades específicas que tenemos que prestar más atención, y el problema de esa atención es el tiempo de la clase" (profesor 04).

La Unidad de Significación: la metodología utilizada por el profesor es un facilitador del aprendizaje al proporcionar recursos tecnológicos y educativos, tales como multimedia, fotos, videos, muestra que los profesores consideran importante la 
disponibilidad de materiales de apoyo en sus clases, como contributivas para el aprendizaje del estudiante.

El uso de recursos tecnológicos en las clases son ahora aliados valiosos en el proceso de enseñanza y aprendizaje. Hay una diversidad de recursos educativos que pueden ayudar a los profesores, entre ellos los tecnológicos, con la participación de TV multimedia, DVD, CD, MP3, data show, softwares/Internet, etc. En este universo, un gran desafío que se presenta a los educadores es elegir, entre los muchos recursos disponibles, los que mejor se ajustan a la naturaleza de sus clases ${ }^{(20)}$.

"(...) comparar (...) mi universidad (...) yo no tenía todo eso, diapositivas, artículos científicos, no era tan fácil como hoy día el estudiante recibe por el eureka" (profesor 05).

"(...) contribuye mucho, desde el momento en que pone un data show que se pasa un video, se pone una foto, es mucho más fácil para el aprendizaje de los estudiantes que sólo escribir en la pizarra" (profesor 10).

Es de destacar que es necesario un profesor cualificado, preparado y seguro, con dominio para hacer frente a esta nueva realidad de los recursos tecnológicos como facilitadores del aprendizaje. Puesto que no son los recursos que transforman las clases de reproducción en clases de construcción, cabe al docente que medie adaptarse la función de los recursos a los objetivos y contenidos de manera que el aprendizaje ocurra.

El artículo 36, de la Ley 9.394/96 LDB - Ley de Directrices y Bases de la Educación anima a la introducción de tecnologías en los diferentes niveles de educación, de modo que "el alumno presente dominio de los principios científicos y tecnológicos que presiden la producción moderna"(21).

La Unidad de Significación: la metodología utilizada por el profesor contribuye al aprendizaje de los alumnos cuando el profesor puede relacionar la metodología apropiada para cada tipo de disciplina y clase, muestra que el docente siente que es necesario relacionar el tema de la clase con la metodología de enseñanza que usará.

Cada disciplina y estudiantes tienen sus propias características, y es por lo tanto difícil proporcionar sugerencias que sean válidas para todos. El método científico no es único, ni sigue siendo el mismo, ya que refleja el contexto histórico y cultural en el que se encuentra. Las metodologías pueden ser adaptadas, modificadas o combinadas por el profesor cuando lo considere apropiado o necesario ${ }^{(22)}$.

"(...) no es posible al profesor trabajar con una metodología única (...) no existe una metodología específica, es en función de la clase en la que se centrará el método de enseñanza" (profesor 06).

"(...) respetar mucho la relevancia y la característica de cada disciplina" (profesor 07).

La Unidad de Significación: la metodología utilizada por el docente no contribuye al aprendizaje de los estudiantes, pues hace falta una participación más activa del estudiante en el proceso de aprendizaje, se refiere al hecho de los profesores a entender que el proceso de enseñanza y aprendizaje sólo es eficaz cuando los 
estudiantes participan activamente y, cuando eso no sucede, no se produce ningún aprendizaje.

El proceso de enseñanza y aprendizaje se compone de cuatro elementos básicos, el profesor, el estudiante, el contenido y las variables ambientales. Entre estos elementos, una de las principales variables que influyen los estudiantes son la buena voluntad, el interés ${ }^{(23)}$.

"Depende, depende de muchas cosas, depende del estudiante, del profesor" (profesor 09).

"(...) creo que todavía hace falta una mayor contribución por parte del alumno

(...) una mayor participación de los estudiantes (...) requiere un mayor dinamismo por los estudiantes (...) y ahí vamos tener un mayor aprendizaje más enfática en el estudiante, tampoco en el maestro. Hoy día, quién más está estudiando es el profesor, en la preparación de sus clases (...) los estudiantes vienen aquí para recibir información" (profesor 14).

La Unidad de Contexto: el (des) conocimiento de la metodología problematizadora por los profesores y la Unidad de Significación: los docentes conocen la metodología problematizadora de manera fragmentada, revela que los profesores consideran conocer la metodología, pero en realidad no saben la forma completa, lo que hace que su comprensión y su aplicación en la práctica docente sea dificultada.

Se observó en el estudio que sólo algunos docentes relataron lo que realmente es la metodología problematizadora y los que más llegaron cerca del real concepto citan pocos pasos de la metodología.

"Ella trabaja en ver lo que el estudiante necesita... ver el punto clave, el conocimiento que el estudiante tiene de la teoría y luego se pone a trabajar las hipótesis" (profesor 03).

"(...) el estudiante trae para usted los problemas y también las soluciones (...) él busca, él va contigo, habla y se ve que el estudiante ha evolucionado" (profesor 04).

La Unidad de Significación: los profesores creen que esta metodología es llevar a cabo estudios de caso en las clases, demuestra que los profesores la confunden con otra metodología que tiene algunas características similares, pero difieren en su totalidad.

El estudio de caso es una metodología de enseñanza, sin embargo, no es la metodología problematizadora. En el estudio de caso, el docente expone el caso a estudiar. En la metodología problematizadora, los problemas son identificados por los estudiantes a través de la observación de la realidad, en la que las preguntas van ocurriendose.

Observada desde diferentes ángulos, la realidad se manifiesta para los estudiantes y profesores con sus características y contradicciones en los hechos concretos y de ahí son extraídos los problemas. La realidad es problematizada por los alumnos. No hay restricciones en cuanto a los aspectos incluidos en la formulación de los problemas, ya que se extraen de la realidad social, dinámica y compleja. 
"Esta metodología es trabajada en estudios de casos" (profesor 01).

"(...) sí, es el estudio de caso (Profesor 05).

"(...) sí, en el tema de estudio de caso" (profesor 06).

"Por lo tanto, es ampliamente utilizado en estudios de casos, por ejemplo" (profesor 11).

La Unidad de Significación: los profesores creen que esta metodología se basa en los problemas, que se no utilizan clases expositivas dialogadas, establece que los profesores entienden que la metodología problematizadora supone problemas, pero aun confunden con otra metodología, el aprendizaje basado en problemas (PBL).

El PBL, iniciales del término Inglés "Problem Based Learning", es una metodología incluida en el conjunto de metodologías activas, se introdujo inicialmente en Brasil en los planes de estudio de Medicina, pero también ha sido experimentada por otros cursos. Se desarrolla basada en la resolución de problemas propuestos, con el fin de que los estudiantes estudien y aprendan determinados contenidos ${ }^{(24)}$.

\section{"(...) se crea un problema para que los estudiantes intenten resolverlo" (profesor 05).}

"(...) se analiza y de alguna manera visualiza los problemas de modo a incorporar en un debate" (profesor 08).

"Es esa de usted trae los problemas de la vida real al interior del aula" (profesor 09).

"Una metodología basada en el problema caso (...) ya no tiene estas clases expositivas" (profesor 12).

A continuación, pasa a describir el alcance del segundo objetivo específico, que era introducir a los profesores la metodología problematizadora. En primer lugar, fue programada con 10 días de antelación una reunión con los 14 profesores que participaron en la encuesta, que se llevó a cabo el 21 de octubre del año 2013, habiendo comenzado a las ocho y terminó a las diez en punto, con 4 profesores presentes.

La reunión se realizó utilizando la metodología problematizadora en los siguientes pasos: $1^{\text {a }}$ fase, observación de la realidad - fueron entregadas noticias a los profesores que reportaban la situación de la educación brasileña y cada uno identificó en ellas problemas relacionados con la educación, siendo la desmotivación de los estudiantes en las aulas la principal; $2^{\mathrm{a}}$ fase, levantamiento de los puntos clave - los profesores levantaron los determinantes de los problemas encontrados y, de los factores determinantes, fue nombrado el uso de metodologías inapropiadas por el profesor; $3^{\text {a }}$ fase, teorización - se les dio a los docentes textos teóricos sobre las diferentes metodologías que fueron citadas por ellos en las entrevistas (estudio de caso, estudio dirigido, seminarios y metodología problematizadora), y cada uno de ellos recibió una metodología distinta para leer. 
A continuación de la teorización, se llegó a la $4^{\text {a }}$ fase de la metodología problematizadora que es el levantamiento de hipótesis de soluciones - los docentes reportaron soluciones para el uso de metodologías inapropiadas, siendo una de ellas la alternancia de las metodologías en cada disciplina, de acuerdo con cada clase. Otra hipótesis para una solución fue la necesidad de una formación en forma de educación continuada para los docentes acerca de las metodologías de enseñanza. Las investigadoras se comprometieron a transmitir esta sugerencia a la coordinación del curso de Enfermería.

En la $5^{\underline{a}}$ y última fase, aplicación a la realidad, cada profesor expresó un ejemplo de uso de la metodología problematizadora en sus clases, y no se observó dificultades por parte de ellos para conocer el uso de la metodología.

Durante la teorización, se observó que los profesores tenían cierta dificultad en diferenciar metodologías, unas de otras, específicamente el estudio de caso del estudio dirigido y la teorización permitió la aclaración de las diferencias entre ellos. En la teorización de la metodología problematizadora se hizo evidente que los profesores sintieron que la metodología estaba siendo aplicada por las investigadoras durante la reunión y que era evidente para todos la aplicabilidad de esta metodología en la práctica docente.

No obstante, al final de la reunión, los profesores señalaron algunas dificultades en el uso de este enfoque en sus clases, siendo una de ellas el mayor tiempo requerido para trabajar cada una de las etapas que en una clase expositiva dialogada. Esta dificultad también se presentará a la coordinación del curso de Enfermería.

Al final, las investigadoras agradecieron la participación de los docentes y ellos se mostraron satisfechos con la reunión.

\section{CONCLUSIÓN}

Identificar los métodos de enseñanza utilizados por los profesores del curso de enfermería permitió darse cuenta de las diferentes metodologías que se utilizan en sus clases y las lagunas conceptuales que los profesores tienen de cada metodología, ya que en el discurso, los profesores no informaron del exacto significado de cada una.

Presentar la metodología problematizadora a los docentes fue un gran desafío para las investigadoras, que se logró con éxito, porque permitió ampliar las perspectivas de los profesores no sólo en esta metodología, sino en las principales metodologías utilizadas por ellos durante las clases. Fue posible responder a sus preguntas acerca de los conceptos, similitudes y diferencias entre las metodologías y, principalmente, abordar y discutir los pasos de la metodología problematizadora y su importancia en la práctica docente junto con otras metodologías.

Cada profesor fue capaz de reflexionar sobre la forma de utilizarlas en su práctica docente y esta presentación y discusión contribuirá a la labor de estos profesores en el curso de Enfermería para conseguir una educación de calidad. En la discusión, se hizo evidente que enseñar y aprender, en la actualidad, necesita ir mucho más allá de transmitir y reproducir el conocimiento. Teniendo en cuenta las nuevas estrategias en materia de educación, se entiende que el profesor debe actuar como mediador, como uno que "muestra el camino", plantea ideas, para que los estudiantes puedan discutir, 
levantar hipótesis, proponer soluciones, siempre considerando su realidad de vida y el contexto en el que se inserta.

Los profesores sugirieron la extensión de esta discusión, llevándola a otros miembros del curso, como una forma de capacitación de profesores. Es de destacar que este entrenamiento está siendo ofrecido por la institución donde se llevó a cabo la investigación y sucederá al final de este año escolar.

\section{REFERENCIAS}

1. BRASIL. Ministério da Educação. Instituto Nacional de Estudos e Pesquisas Educacionais Anísio Teixeira. Censo da educação superior 2010 [Internet]. Acesso em: $20 \quad$ Set 2013.2 Disponível em: http://webcache.googleusercontent.com/search?q=cache:vtrtllz8 YEJ:portal.mec.gov. br/index.php\%3Foption\%3Dcom docman\%26task\%3Ddoc download\%26gid\%3D933 2\%26ltemid $+\& c d=5 \& h l=p t-B R \& c t=c l n k \& g l=b r$.

2. Costa JS. Docência no ensino superior: professor aulista ou professor pesquisador. Caderno Discente do Instituto Superior de Educação. 2008;2(2):41-61.

3. Silva RM, Silva ICM, Ravalia RA. Ensino de Enfermagem: reflexões sobre o estágio currcular supervisionado. Rev Práxis.2009;1(1):37-41.

4. Silva EM, Moarais MJB. Avaliação da Aprendizagem: pressupostos ideológicos de uma prática de avaliação na Educação de Jovens e Adultos [dissertação na Internet]. Belém (PA): Universidade da Amazônia; 2002. Acesso em: 23 Out 2013. Disponível em:

http://www.nead.unama.br/site/bibdigital/monografias/avaliacao da aprendizagem.pdf

5. Rangel M. Métodos de ensino para a aprendizagem e a dinamização das aulas. $3^{\text {a }}$ ed. Campinas: Papirus; 2007.

6. Vilarinho LRG. Didática: Temas Selecionados. Rio de Janeiro: Livros Técnicos e Científicos; 1985.

7. Evangelista YSP, Chaves EV. Ensino de química: uma realidade concreta do saber. In: 3ํㅡㄹ Econtro Nacional de Ensino de Ciências da Saúde e do Meio Ambiente; 2012 Maio [Internet]. Niterói (RJ), Brasil. Niterói: Universidade Federal Fluminense (UFF); 2012. Acesso em: 12 Set 2013. Disponível em: http://www.ensinosaudeambiente.com.br/eneciencias/anaisiiieneciencias/trabalhos/T6 2.pdf.

8. Pereira ALF. As tendências pedagógicas e a prática educativa nas ciências da saúde. Cad. Saúde Pública [Internet]. 2003;19(5):1527-34. Acesso em: 13 Mar 2013. Disponível em: http://www.scielo.br/pdf/csp/v19n5/17825.pdf.

9. Schaurich D, Cabral FB, Almeida MA. Metodologia da problematização no ensino Em enfermagem: uma reflexão do vivido no profae / RS ${ }^{a}$. Esc Anna Nery. 2007;11(2):319-24.

10. Ribeiro DM, Rauen MS, Prado ML. O uso da Metodologia Problematizadora no ensino em odontologia. Rev Odont Univ Cid São Paulo. 2007;19(2):217-21.

11. Neves JL. Pesquisa qualitativa-caracteristicas, uso e possibilidades. Cad Pesq Adm [Internet]. 1996;1(3):1-5. Acesso em: 04 Mar 2013. Disponível em: http://www.ead.fea.usp.br/cad-pesq/arquivos/c03-art06.pdf.

12. Cervo AL, Bervian PA, Silva R. Metodologia científica. 6. ed. São Paulo: Pearson Education do Brasil; 2007.

13. Boni V, Quaresma SJ. Aprendendo a entrevistar: como fazer entrevistas em Ciências Sociais. Em Tese. 2011;2(1):75.

14. Bardin L. Análise de Conteúdo. 1ª ed. Lisboa (Pt)l: Edições 70; 1994. 
15. Mozzato AR, Grybovski D. Análise de conteúdo como técnica de análise de dados qualitativos no campo da administração: potencial e desafios. RAC [Internet]. 2011;15(4):731-47. Acesso em: 17 Set 2013. Disponível em: http://www.scielo.br/pdf/rac/v15n4/a10v15n4.pdf.

16. Graham A. Como escrever e usar estudos de caso para o ensino e aprendizagem no setor público. $1^{\underline{a}}$ ed. Brasília: ENAP; 2010.

17. Scarpato M. Didática na Prática, Os Procedimentos de Ensino Fazem a Aula Acontecer. 1를 ed. São Paulo: Avercamp; 2004.

18. Costa MNMG, Araújo RP. A importância da visita técnica como recurso didático metodológico. Um relato na prática do IF Sertão Pernambucano. In: Anais do 5o Congresso Norte Nordeste de Pesquisa e Inovação. Palmas (TO); 2012.

19. Osti A. As dificuldades de aprendizagem na concepção do professor [Dissertação na Internet]. Campinas (SP): Universidade Estadual de Campinas; 2004. Acesso em: 11 Out 2013. Disponível em: http://www.bibliotecadigital.unicamp.br/document/?code=vtls000321202\&fd=y.

20. Viscovini RC, Gozzi ME, Arias C, Miranda DP, Sigoli LSM, Zanqueta VA. Recursos pedagógicos e atuação docente [Internet]. In: Anais do 9o Congresso Nacional de Educação EDUCERE. Curitiba; 2009. Acesso em: 25 Set 2013. Disponível em: http://www.pucpr.br/eventos/educere/educere2009/anais/pdf/1872_1130.pdf. 21. Brasil. Lei no 9.394, de 20 de dezembro de 1996 [Internet]. Brasília,1996. Acesso em: 22 Out 2013.2 Disponível http://legis.senado.gov.br/legislacao/ListaTextolntegral.action?id=75723.

22. Marsulo MAG, Silva RMG. Os métodos científicos como possibilidade de construção de conhecimentos no ensino de ciências. Revista Electrónica de Enseñanza de las Ciencias. 2005;4(3):30.

23. Santos SC. O processo ensino-aprendizagem e a relação professor- aluno: Aplicação dos "sete princípios para a boa prática na educação de ensino superior". Cad Pesq Adm [Internet]. 2001;8(1):70-3. Acesso em: 22 Set 2013. Disponível em: http://www.regeusp.com.br/arquivos/v08-1 art07.pdf.

24. Berbel NAN. As metodologias ativas e a promoção da autonomia de estudantes. Semina: Ciências Sociais e Humanas [Internet]. 2011;32(1):30-3. Acesso em: 27 Set 2010. Disponível http://www.proiac.uff.br/sites/default/files/documentos/berbel_2011.pdf.

Recibido: 21 de noviembre de 2013; Aceptado: 16 de enero de 2014

ISSN 1695-6141

๑ COPYRIGHT Servicio de Publicaciones - Universidad de Murcia 